\title{
Migración calificada entre México-Estados Unidos. Desafíos y opciones de política
}

\author{
RODOLFO TUIRÁN* \\ JOSÉ LUIS ÁVILA**
}

\begin{abstract}
RESUMEN: Este artículo contiene estimaciones del volumen de la migración calificada de México a Estados Unidos en el periodo 2000-2012, así como de los connacionales con esa característica que, después de residir un tiempo en el país vecino del norte, regresaron a México en el segundo quinquenio de la década pasada; se delinean, para ambos flujos de migrantes, sus perfiles sociodemográficos, laborales y económicos. Considerando la evolución reciente y creciente importancia de este fenómeno, se revisan críticamente las principales propuestas de conceptualización, las líneas de política migratoria y su pertinencia para países como México. Por último, se formula una agenda de investigación en la materia, incluyendo el impacto de la migración calificada en la capacidad nacional de innovación, al tiempo que se sugieren algunas opciones de política para que la migración internacional de talentos no se traduzca en una pérdida definitiva de capital humano, sino en una oportunidad para el desarrollo nacional. PALABRAS CLAVE: migración calificada, desarrollo económico, ciencia, tecnología, México-Estados Unidos.
\end{abstract}

ABSTRACT: This article estimates the volume of skilled migration from Mexico to the United States in the period 2000-2012, including those who, after living for a time in the neighboring country to the north, returned to Mexico in the latter half of the last decade; it outlines, for both types of migratory flows, their socio-demographic, employment and economic profiles. Taking into account the recent evolution and growing importance of this phenomenon, the article critically reviews the proposed principles of its conceptualization, the trends of migration policy and their relevance for countries like Mexico. Lastly, it proposes a research plan on this topic, including the impact of skilled migration on the nation's capability for innovation, while suggesting policy options for international skilled migration, which rather than leading to the permanent loss of human capital, could represent an opportunity for national development.

KEY WORDS: skilled migration, economic development, science, technology, Mexico-United States.

* Subsecretario de Educación Media Superior de la Secretaría de Educación Pública (SEP), México.

** Profesor de la Facultad de Filosofía y Letras de la Universidad Nacional Autónoma de México (UnAM), México.

Los autores agradecen al Act. Carlos Fuentes Villalba su colaboración en este artículo. 


\section{INTRODUCCIÓN}

$\mathrm{E}$ 1 ascenso de la migración internacional calificada es uno de los fenómenos globales de nuestro tiempo: expresa el cúmulo de retos y oportunidades que plantea la dinámica del sistema global a las naciones en vías de desarrollo. Si hace medio siglo solía reconocerse como "fuga de cerebros», por diversos factores — como la intensificación de este fenómeno desde 1990, los cada vez mayores vínculos de los países de origen con su respectiva diáspora y el retorno de numerosos talentos a sus países de origen - alientan hoy la opinión de que este fenómeno puede ser convertido en «circulación de talentos» (brain circulation). Esta transformación implica pasar de un juego de suma cero, en el que pierden los países emisores y ganan los receptores, a otro de suma positiva, en el que todas las naciones ganan capacidades y habilidades de innovación, que hoy en día son los activos más valiosos del desarrollo en la emergente sociedad del conocimiento.

Cabe destacar, sin embargo, que esa percepción optimista de la migración internacional calificada es tan sólo una posibilidad frente a la que se interpone una variedad amplia de obstáculos y condicionantes de muy diversa índole, que las políticas migratorias por sí solas no pueden superar. Por ejemplo, para cristalizar las promesas de la circulación de talentos es necesario, entre otras cuestiones relevantes, que las naciones de origen de la migración calificada vinculen la política migratoria con sus estrategias nacionales de desarrollo - lo que incluye estructurar sólidos sistemas nacionales de ciencia, tecnología e innovación-, y sumen energías y voluntades para lograr un consenso en los organismos multilaterales y de gestión global sobre una nueva gobernabilidad de las migraciones internacionales, cuyo curso hasta ahora ha sido determinado tanto por las asimetrías de poder en el sistema mundial como por la lógica de la acumulación de capital a escala global, hechos que favorecen a las naciones de mayor desarrollo.

Desde la perspectiva de las naciones emergentes, un punto de partida ineludible en la reflexión crítica y el diseño de la nueva gobernabilidad de la migración internacional calificada es el de asumir un enfoque de responsabilidad compartida y una visión de mediano y largo plazos que armonicen 
los intereses - a menudo contrapuestos-y sustenten la cooperación entre las naciones. Las políticas unilaterales no pueden regular un fenómeno dinámico y complejo cuyas causas y consecuencias se localizan en los países de origen y destino de la migración internacional calificada.

Como se comprenderá, la intensificación de la migración calificada hace necesario contribuir a dar respuesta a preguntas como las siguientes: ว̇cuáles son sus dimensiones, modalidades y características?, żcómo se articulan las diferentes expresiones de este fenómeno con el proceso global de las migraciones internacionales?, żcuáles son las principales causas y consecuencias de la migración calificada?, y żqué tipo de políticas y acciones concretas son las más recomendables o más promisorias para encarar este fenómeno?

En este artículo concentramos la atención en la migración calificada que se origina en México, el país con el mayor número de emigrantes de este tipo en América Latina y el Caribe. En la primera sección examinamos brevemente las tendencias mundiales de la migración calificada, configuradas por la expansión económica ocurrida entre 1990 y 2007, y más tarde por la crisis global de 2007 a 2008 con la recesión que le sucedió. En la segunda sección analizamos la corriente migratoria internacional de personas altamente calificadas entre México y Estados Unidos durante la última docena de años, precisando tanto las tendencias y características básicas de los profesionistas y posgraduados mexicanos que viven en el vecino país del norte, como de los que retornan al país. En la tercera sección se reflexiona sobre algunas propuestas teóricas sobre la migración internacional calificada y se revisa su pertinencia para el caso mexicano. El artículo cierra con la identificación de algunas opciones de política para que la migración internacional calificada no se traduzca en una pérdida definitiva de capital humano, sino en una oportunidad para el desarrollo de los países emisores como México.

\section{ASCENSO DE LA MIGRACIÓN INTERNACIONAL CALIFICADA}

Resulta ya un lugar común decir que vivimos en un mundo donde el saber científico y técnico adquiere centralidad en los diferentes ámbitos de la vida 
económica, social y cultural. La generación y aplicación de dicho conocimiento ha impuesto a la sociedad una nueva dinámica en la que interactúan las universidades y centros de investigación con empresas, laboratorios y agencias gubernamentales, entre otros actores relevantes.

Los países se distinguen entre sí, cada vez más, por la capacidad que tienen tanto de incorporar conocimiento a todos los sectores y ramas de la economía como de involucrar en esa tarea a un volumen creciente de personas altamente calificadas. La creciente demanda de talentos ha contribuido a intensificar la migración internacional de recursos humanos especializados, en respuesta a las nuevas oportunidades, incentivos y recursos que genera la economía del conocimiento.

Las economías más avanzadas conforman los principales centros de innovación y del desarrollo científico y tecnológico y operan como poderosos imanes que atraen cuantiosos flujos de especialistas capaces de generar ideas o productos, así como de aplicar conocimiento en complejos procesos de producción e innovación. En términos generales, las personas con altas calificaciones son atraídas por los países avanzados porque ofrecen mayores oportunidades de encontrar un empleo apropiado a sus capacidades, un salario más elevado, infraestructura científica y profesional y un ambiente institucional propicio. ${ }^{1}$ También tienen enorme importancia las facilidades de integración social que ofrecen los gobiernos de los países de destino a los migrantes calificados, así como las oportunidades educativas y de salud para el desarrollo de sus familias (Docquier y Rapoport, 2011; De Haas, 2010).

De acuerdo con estimaciones de la Organización de las Naciones Unidas (ONU), el número de migrantes con estudios de licenciatura y posgrado de 25 años o más de edad, residentes en países de la Organización para la Cooperación y el Desarrollo Económicos (OCDE):

${ }^{1}$ Un rasgo distintivo del patrón de la migración internacional calificada es que su destino principal continúan siendo las naciones de mayor desarrollo. Tan sólo en la última década del siglo pasado un tercio de esos migrantes se dirigía de los países emergentes a Estados Unidos; otro tercio de esas naciones hacia Europa; y el resto corresponde a intercambios poblacionales entre las propias naciones en vías de desarrollo (OIM, 2009).

$46 \frac{\text { SEGUNDO SEMESTRE } 2013}{\text { MIGRACIÓN Y DESARROLLO NÚM. } 21}$ 
- Aumentó 67\% entre 1990 y 2000 al pasar de 12 a 20 millones de personas. Este flujo de migrantes calificados explica casi la mitad del aumento total de la inmigración de personas de ese amplio grupo de edad a los países de la OCDE.

- La migración calificada hacia los países de la OCDE continuó creciendo entre 2000 y 2007, al pasar de 20.3 a 28.5 millones de personas, lo que representa un aumento de 40\% (oIm: 2009: 14).

Las regiones en desarrollo desempeñaron en ambos periodos un papel protagónico en la migración internacional calificada: América Latina y el Caribe es la región del mundo que en el periodo 1990-2007 registró el mayor crecimiento relativo de la migración calificada (véase gráfica 1), con un aumento de $155 \%$, seguida de cerca por África (152\%) y Asia (145\%) (OIM, 2009).

La crisis económica mundial de 2007-2008 redujo el ritmo de crecimiento de la inmigración a los países de la OCDE. ${ }^{2}$ La crisis afectó más a los migrantes con baja calificación. ${ }^{3}$ El impacto más adverso de la crisis en esos países ha sido para los migrantes de América Latina y del norte de África. ${ }^{4}$

En los años recientes volvieron a aumentar los flujos de inmigración hacia esos países, aunque todavía se mantienen muy por debajo de los niveles anteriores a la crisis global. Las naciones de destino están realizando ajustes a sus políticas de inmigración para favorecer el ingreso de los que poseen las más altas calificaciones profesionales.

${ }^{2}$ Por ejemplo, la inmigración legal permanente hacia los países de la oCDE bajó 6\% en 2008, luego de cinco años en que había registrado un crecimiento promedio anual de 11\%; en 2009, esa disminución en los flujos continuó y se extendió a casi todos los países.

${ }^{3}$ La tasa de desempleo promedio de los extranjeros aumentó 5 puntos porcentuales entre 2008 y 2012, en comparación con 3 puntos porcentuales para los nacionales; el desempleo prolongado de los migrantes se está convirtiendo en un reto grave para muchos países de la OCDE. En 2012, casi uno de cada dos migrantes desempleados había buscado trabajo durante más de un año.

${ }^{4}$ Por ejemplo, los migrantes del norte de África en Europa enfrentaron una tasa de desempleo récord, de $26.6 \%$, en 2012. 


\section{GRÁFICA 1}

Número de migrantes calificados en los países de la OCDE y tasa de emigración según región de origen, 1990, 2000 y 2007
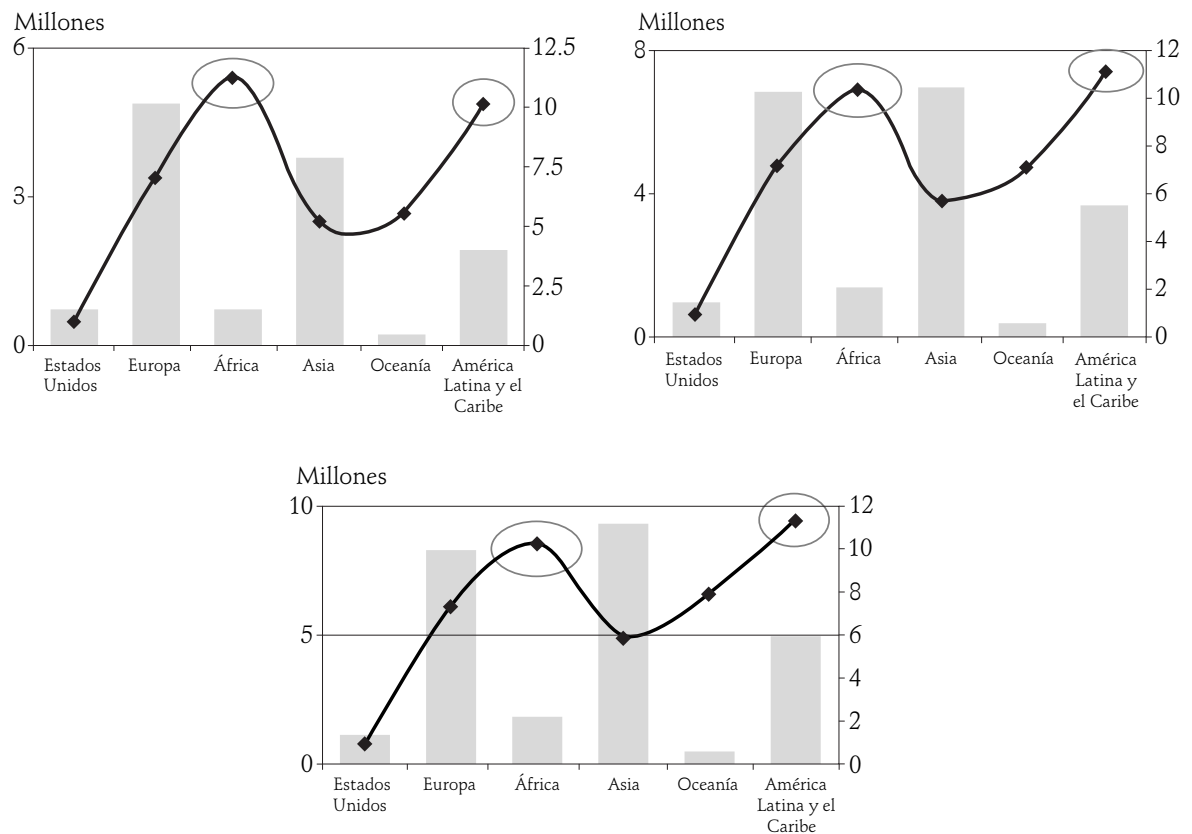

Nota: Los flujos más cuantiosos de migrantes altamente calificados ocurren desde los países del sur a los países del Norte, destacando Estados Unidos como el primer país de destino.

Fuente: OIM, La emigración de recursos humanos calificados desde países de América Latina y el Caribe, oIm, Venezuela, 17 de junio de 2009, p. 20.

\section{DINÁMICA Y PERFIL DE LA MIGRACIÓN CALIFICADA eN el CORRedor México-Estados Unidos}

México es el país latinoamericano con más migrantes internacionales altamente calificados en los países de la OCDE, mayoritariamente en Estados Unidos. Entre los rasgos más sobresalientes de este movimiento migratorio, sobresalen los siguientes:

1. El número total de migrantes altamente calificados nacidos en México y residentes en Estados Unidos creció 2.4 veces entre 2000-2012, al pasar de

$48 \frac{\text { SEGUNDO SEMESTRE } 2013}{\text { MIGRACIÓN Y DESARROLLO NÚM. } 21}$ 
411 mil a 1 millón 15 mil personas. Esta última cifra representa poco menos de uno de 10 connacionales en Estados Unidos (véase gráfica 2).

2. Cabe precisar que a la par del fenómeno migratorio general, la migración de personas altamente calificadas tuvo un crecimiento acelerado al comienzo del nuevo siglo, y se ha contenido en años recientes. Así, el aumento neto por año en 2000-2005 ascendió a 61 mil migrantes con licenciatura y a $11 \mathrm{mil}$ con posgrado; en el siguiente lustro las cifras se ubicaron en 65 y siete mil, y en el trienio 2010-2012 descendieron a 13 mil y 1 mil, respectivamente.

3. Del número de migrantes mexicanos altamente calificados residentes en Estados Unidos en 2012, un total de $862 \mathrm{mil}$ (84.9\%) posee estudios del nivel profesional (licenciatura, técnico superior universitario y profesional asociado); el resto (153 mil) tiene un posgrado (15.1\%) (véase gráfica 2). Las cifras representan para México una proporción muy significativa de los mexicanos con mayor capital humano: uno de cada 10 mexicanos con título profesional y poco más de uno de cada cuatro con posgrado vive en Estados Unidos. Además, conviene precisar que alrededor de la mitad de los mexicanos calificados realizó estudios en México y el resto en Estados Unidos, lo que revela los estrechos vínculos de la migración calificada con otras modalidades migratorias entre ambos países.

4. La emigración calificada combina diferentes tipos de flujos. Incluye los movimientos de estudiantes, científicos y académicos; también los de quienes se desempeñan en ocupaciones ejecutivas y de gestión, así como los de técnicos y profesionales que no encuentran empleo en México y que incluso buscan insertarse en ocupaciones no profesionales en Estados Unidos, entre otros. Algunos de estos flujos tienen un carácter más o menos permanente o de largo aliento; otros, en cambio, implican movimientos circulares y proyectos transitorios, lo que impone en cada situación complejos desafíos en materia de políticas públicas.

5. De las características sociodemográficas de los connacionales con altas calificaciones que viven en Estados Unidos, destaca la menor edad respecto de los nativos estadounidenses con esa escolaridad y de los inmigrantes de otros países. ${ }^{5}$

${ }^{5}$ Alrededor de $35 \%$ de quienes poseen títulos profesionales o de posgrado tienen menos de 35 años de edad (entre los nativos y otros inmigrantes las proporciones son de 26.4 y $33 \%$, respectivamente). En cambio, $37.3 \%$ de las y los mexicanos con esa escolaridad tiene 45 o 


\section{GRÁFICA 2}

Población mexicana residente en EEUU con estudios superiores concluidos por nivel educativo, 2000-2012

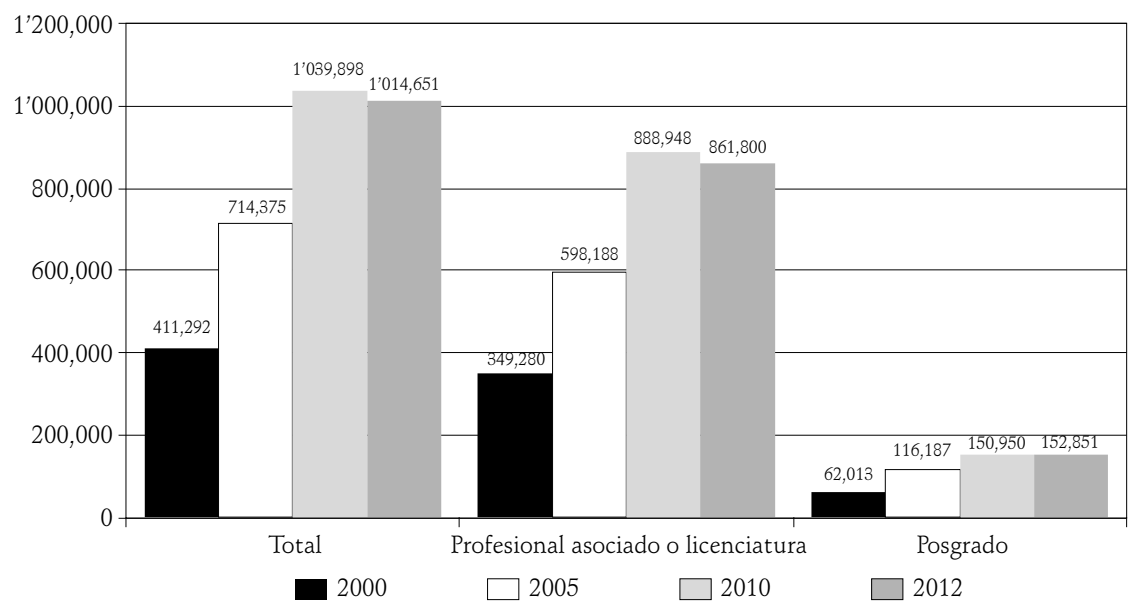

Fuente: Estimaciones propias con base en el Bureau of Census, Current Population Survey (CPS), suplemento de marzo, 2000, 2005, 2010 y 2012.

6. La composición por sexo de la población altamente calificada residente en Estados Unidos muestra una presencia ligeramente mayor de las mujeres, tanto entre la migración mexicana (50.6\%), como entre la inmigración de otros países (52.2\%). Considerando las tendencias imperantes en décadas pasadas, estos parámetros describen tanto el logro educativo de las mujeres, como el hecho de que para ellas la migración es una opción tan importante como para los varones.

7. En cambio, los mexicanos altamente calificados que viven en el vecino país del norte tienen una participación desventajosa en el mercado de trabajo tanto con relación a la población nativa, como a otros inmigrantes. Entre los rasgos de este movimiento, destacan los siguientes:

- La participación económica de los migrantes mexicanos es muy heterogénea: laboran tanto en unidades productivas y de servicios, como también en centros de investigación e instituciones educativas.

más años de edad (entre los nativos se eleva hasta $54.2 \%$ y a uno de cada tres entre los inmigrantes oriundos de otros países).

$50 \frac{\text { SEGUNDO SEMESTRE } 2013}{\text { MIGRACIÓN Y DESARROLLO NÚM. } 21}$ 
- Alrededor de $45 \%$ se desempeña en ocupaciones profesionales y de servicios, $12.7 \%$ en ocupaciones gerenciales, financieras y de negocios, y $12.2 \%$ en ocupaciones de apoyo administrativo; a distancia le siguen las ocupaciones de la construcción y minería (7.8\%), de la producción (6.7\%) y las relacionadas con el transporte $(5.0 \%)$.

- Los salarios de los inmigrantes mexicanos altamente calificados son significativamente inferiores no sólo a los percibidos por los nativos estadounidenses con credenciales académicas equivalentes, sino también al de los inmigrantes de otros países. Así, el ingreso promedio anual de la población ocupada con nivel de profesional y posgrado es de 65 mil dólares para los nativos, 67 mil dólares para otros inmigrantes y de casi 45 mil dólares para los nacidos en México.

- Los mexicanos con nivel de licenciatura ganan el equivalente a 74 y $75 \%$ del ingreso de los nativos estadounidenses y de otros inmigrantes, respectivamente.

- Alrededor de $13 \%$ de los connacionales altamente calificados se encuentra en situación de pobreza (de acuerdo con la norma estadounidense), en contraste con sólo $5.3 \%$ de los nativos y $9.0 \%$ de otros inmigrantes.

- Vista la retribución salarial desde el otro extremo, destaca que $76.1 \%$ de los migrantes mexicanos calificados tiene un ingreso $50 \%$ o más del valor de la línea de la pobreza, y en el caso de los nativos la proporción se eleva hasta 90.6 y a $86.0 \%$ entre los inmigrantes.

\section{El difícil retorno a México}

El flujo de ida de la migración mexicana suele estar acompañado de otro, poco conocido, en sentido contrario. Desde el punto de vista de la escala de los movimientos de ida y de regreso, conviene tener presente que si bien México ocupa el sexto lugar a nivel mundial con más migrantes internacionales altamente calificados, ese posicionamiento no se corresponde con la escala de la migración de retorno.

De acuerdo con el más reciente censo de población de México, en 20052010 regresaron a nuestro país, provenientes de Estados Unidos, alrededor 
de un millón de personas, de las cuales sólo 61 mil (5.6\%) poseían alta escolaridad. Esto representa 40 mil profesionistas, 16 mil maestros y poco más de cinco mil doctores. Las características de estos migrantes ilustran la índole de los problemas y características nacionales para hacer de la migración de retorno una estrategia para la promoción del desarrollo científico y tecnológico del país, así como de la capacidad de innovación.

Del total de los migrantes altamente calificados que después de permanecer un tiempo en Estados Unidos regresaron a México entre 2005 y 2010, destacan las siguientes características:

- Casi seis de cada 10 (58\%) migrantes son varones y la gran mayoría tiene entre 30 y 45 años de edad. Un total de 74\% forma parte de la población económicamente activa (PEA) y la gran mayoría (95\%) está ocupada en el año del levantamiento censal.

- En su mayor proporción, la posición en el trabajo corresponde a trabajadores remunerados (72\%), 16.5\% trabaja por cuenta propia y sólo $9.6 \%$ es empleador.

- Asimismo, destaca que entre las ocupaciones principales, 49\% son profesionistas y técnicos y $22.8 \%$ funcionarios y directores; el resto se distribuye en otras ocupaciones.

- La mayoría trabaja en el sector servicios (52.2\%), seguido por el comercio (13.3\%), la construcción (12.4\%) y finalmente en el gobierno y los organismos internacionales (7.3\%).

El perfil laboral de los migrantes de retorno revela el gran reto que significa para México que la migración internacional calificada no se traduzca ni en una pérdida definitiva (brain drain) ni en un desperdicio de talentos (brain waste). Las políticas en esta materia, dirigidas a maximizar los beneficios y minimizar los costos de la migración calificada, deberán considerar en su diseño e instrumentación muy diversos factores.

Es escaso el conocimiento sobre los costos y los beneficios de la migración calificada de mexicanos a Estados Unidos. No se dispone de una estimación precisa del costo de la migración calificada, que considere, entre otros aspectos, los recursos que el Estado y la sociedad han destinado a la alimentación, la educación y el cuidado de la salud de los migrantes. Tampoco se 
cuenta con evaluaciones de los costos de oportunidad directos - el valor de producción que no se realiza por la emigración — e indirectos — el costo del potencial de desarrollo e innovación que se pierde con la «fuga de cerebros» ni de los impactos fiscales de la migración calificada (Knerr, 2007).

Un análisis equilibrado de los costos del fenómeno de la migración calificada también exige reconocer los beneficios que incorporan al país los migrantes de retorno, como son las remesas de dinero, las inversiones de los migrantes y de sus organizaciones sociales en la construcción de infraestructura y equipamiento de sus comunidades de origen, las inversiones productivas, la creación de patrimonio, el ahorro y el desarrollo de habilidades de innovación. ${ }^{6}$

Sólo como una indicación, estudios recientes estiman que México habría ahorrado al país vecino por el gasto en la educación de los migrantes calificados y no calificados alrededor de 81 mil millones de dólares durante el periodo 1994-2008. Se trata de un volumen promedio de recursos de cerca de 6 mil millones de dólares por año, lo que equivale a poco más de medio punto porcentual del producto interno bruto (PIB) de México (Albo y Ordaz, 2011).

El monto de remesas familiares, que es el dividendo más directo e inmediato de la migración en todas sus modalidades, permite a su vez aproximarnos a uno de sus beneficios más visibles. En el periodo referido, nuestro país recibió por ese concepto un total de 185 mil millones de dólares, es decir, por cada dólar que México gastó en la educación de los migrantes, recibió poco más de dos dólares en el periodo 1994-2008 (Albo y Ordaz, 2011).

Estas cifras sugieren que México estaría haciendo de la migración una empresa rentable, pero si se consideran los demás costos directos de la migración ya aludidos, como los invertidos en la alimentación y el cuidado de la salud de los migrantes, así como la pérdida del potencial de desarrollo por fuga de capital humano, las cuentas no serían tan optimistas.

En todo caso, la estimación es tan sólo indicativa de los profundos impactos que el fenómeno de la migración internacional calificada tiene en la

${ }^{6}$ Una reflexión más amplia — que incluye varias estimaciones- de los costos y beneficios de la migración, incluidas algunas contribuciones de los connacionales a la sociedad estadunidense, puede verse en Cypher y Delgado (2012). 
economía y la sociedad mexicana, y sirve para llamar la atención acerca de un asunto tan relevante de la agenda nacional.

\section{¿De la fuga a la circulación de talentos?}

Han sido muy diversas y diferenciadas (según los tipos de migrantes) las políticas migratorias aplicadas por las naciones de destino, a raíz de la severa disminución del piB y sus efectos adversos en los mercados de trabajo detonados por la crisis económica global de 2007-2008 y la recesión que le sucedió. En la comunidad europea, por ejemplo, las políticas de inmigración se han vuelto más restrictivas en la contratación de extranjeros para proteger su fuerza laboral ante el creciente desempleo (Marmolejo, 2009), lo cual alcanza incluso a la migración calificada. ${ }^{7}$

Sin embargo, al mismo tiempo se ha empezado a utilizar en la Comunidad Europea un sistema de puntos para favorecer la aceptación de inmigrantes más calificados. Tan sólo en 2011 y 2012, siete países de la OCDE modificaron sus sistemas para atraer a sus mercados laborales a inmigrantes altamente calificados, así como a estudiantes internacionales titulados (Cfr. EUR-Lex, 2009), y se han fortalecido los programas para atraer inversionistas y empresarios. ${ }^{8}$

La política migratoria ha tenido una trayectoria diferente en Estados Unidos, el epicentro de la crisis global y principal destino de la migración internacional. ${ }^{9}$ En medio de esta coyuntura económica adversa, la élite polí-

7 Por ejemplo, «los Estados miembros (de la Unión Europea) han tratado de manera diferente a la crisis económica. Algunos [...] han liberalizado las políticas (como Suecia) [...] Sin embargo, la mayoría [...] han aplicado políticas más restrictivas para los trabajadores altamente cualificados» (Cerna, 2010: 9).

8 A respecto destaca la Tarjeta Azul EU que la Comunidad Europea adoptó en 2009, la cual permite trabajar durante un máximo de cuatro años a personas altamente calificadas procedentes de países extracomunitarios que cuentan con una oferta de empleo.

${ }^{9}$ Aun cuando ahí la política económica ha sido más eficaz para moderar el impacto de la crisis, el desempleo sigue siendo elevado — afecta más a la población inmigrante-y los trabajadores han visto disminuir su nivel de vida, e incluso la desigualdad ha alcanzado niveles preocupantes (Stiglitz, 2012).

$\mathbf{5 4} \frac{\text { SEGUNDO SEMESTRE } 2013}{\text { MIGRACIÓN Y DESARROLLO NÚM. } 21}$ 
tica y la sociedad civil estadounidense han otorgado a la política migratoria una importancia y un enfoque contrastante al conferido por las autoridades de la Comunidad Europea. En particular, llaman la atención tanto el lugar tan relevante que el presidente estadounidense asignó a la política de inmigración en la campaña por un segundo periodo de gobierno (2012), como las acciones consecuentes con ese compromiso. Sin duda, esta opción de política es una señal alentadora para los migrantes.

Si bien la iniciativa de reforma migratoria estadounidense está dirigida principalmente a ofrecer un camino para regular el flujo no autorizado —en su gran mayoría formado por trabajadores no calificados-, no hay duda de que tendrá también profundas implicaciones para la migración calificada. ${ }^{10}$ De hecho, en las disposiciones del Senado contenidas en la iniciativa de reforma migratoria es evidente la convicción de que el reclutamiento de los inmigrantes altamente calificados propicia, como lo señala el informe de la Casa Blanca, que "la nación eventualmente esté bien posicionada en la competencia global por las nuevas ideas, los nuevos negocios, y los empleos del futuro».

Como se advierte, las políticas migratorias que están aplicando los países de destino de la migración de personas altamente calificadas han contribuido a reabrir el debate sobre los costos y beneficios de este fenómeno. Desde los años sesenta, dicho debate se desplazó como un péndulo de una posición pesimista hacia otra más optimista (De Hass, 2010).

La «fuga de cerebros» y la posterior diversificación de la migración calificada

Los países en desarrollo han conceptualizado tradicionalmente la migración internacional calificada permanente como una «fuga de cerebros». De acuerdo

${ }^{10}$ De acuerdo con la iniciativa que aprobó una de las comisiones del Senado el pasado 21 de mayo, la nueva ley aumentaría las llamadas Visas H-1b, de 65 mil a 110 mil, con la posibilidad de crecer a 180 mil, y ampliaría las facilidades de las compañías tecnológicas para contratar a trabajadores extranjeros calificados. 
con este punto de vista, el fenómeno implica no sólo la pérdida de recursos invertidos por la sociedad y el Estado en la educación de sus trabajadores, sino que también significa una merma del capital humano y del potencial de desarrollo.

En consecuencia, la migración calificada ha sido vista como un juego de suma cero: las naciones de origen pierden las competencias, las destrezas y las potencialidades de innovación de los migrantes calificados y las naciones de acogida las ganan. Este enfoque sustentó la inclinación de los países emisores hacia políticas contrarias al laissez-faire en el mercado internacional de trabajadores calificados. ${ }^{11}$

En el contexto latinoamericano, esas valoraciones fueron coherentes con las teorías del desarrollo más influyentes, sobre todo en la década de los cincuenta y sesenta (estructuralismo, desarrollismo y marxismo, entre otras). ${ }^{12}$

Más tarde, la intensificación de la internacionalización del capital productivo, primero con las expansión de las llamadas empresas transnacionales, y más tarde a través de la fábrica mundial — que segmenta en varias naciones el ciclo de producción de un bien-, trastocó la división internacional de trabajo e impulsó el tránsito hacia una nueva etapa del sistema mundial conocida como global. En ella, el conocimiento se transformó en el activo más valioso del desarrollo.

La dinámica de la economía global que se afianza en la última década del siglo pasado trastocó las pautas tradicionales de la migración de personas

${ }^{11}$ La Conferencia de las Naciones Unidas sobre Comercio y Desarrollo, celebrada en Santiago en 1972 reconoció el fenómeno de fuga de cerebros como una «transferencia inversa de tecnología».

12 Por ejemplo, la fuga de cerebros de América Latina hacia Estados Unidos podía ser explicada desde el enfoque de la dependencia tecnológica de la región respecto de los países centrales, quienes no sólo concentraban el progreso técnico, sino que incluso no lo propagaban hacia los países periféricos, reproduciendo y ampliando la brecha tecnológica entre el centro y la periferia del sistema mundial (Prebisch, 1949; Dos Santos, 1968). Desde esa perspectiva, la compra de tecnología en los países avanzados, el pago de sus servicios y la fuga de cerebros — que inhibe procesos de "asimilación creativa» de tecnología importada - fueron conceptualizados como manifestaciones de un proceso más amplio de intercambio desigual entre el centro y periferia del sistema mundial (Emmanuel, 1971; Prebisch; 1981).

$56 \frac{\text { SEGUNDO SEMESTRE } 2013}{\text { MIGRACIÓN Y DESARROLLO NÚM. } 21}$ 
altamente calificadas. Desde entonces no sólo aumentó su cuantía a una velocidad inédita, sino que se diversificaron las modalidades migratorias y se transformaron los patrones de origen y destino.

Junto al impulso de la migración permanente de personas altamente calificadas fue cobrando significación la migración temporal (brain exchange) y de retorno, y con ello se fueron acumulando algunas evidencias de la transferencia de conocimientos (codificado y tácito) y de habilidades de innovación de los países de destino hacia los de origen de la migración internacional, al tiempo que el flujo de remesas alcanzó valores sin precedente.

Asimismo, los procesos de integración económica mundial y el desarrollo de sistemas de innovación globales intensificaron la movilidad internacional de talentos (brain circulation). En algunos casos mediante típicos desplazamientos dentro de una misma corporación o entre las empresas subcontratadas en los países emergentes (outsourcing); en otros casos, como parte de estrategias de relocalización de empresas globales mediante enclaves de alta tecnología en otras naciones; o también como respuesta a la mayor demanda mundial de talentos y el influjo de las redes globales del conocimiento.

La movilidad internacional también se intensificó entre la población estudiantil — que para realizar estudios de posgrado se dirige a la ciudades globales, principalmente de Estados Unidos- así como del personal académico, tanto a través de estancias cortas de investigación como en la formación de redes del conocimiento. Fructificaron también las alianzas y consorcios internacionales de las instituciones de educación superior y centros de investigación.

\section{OpCiONES DE POLÍtica}

La escalada de la migración internacional calificada y la diversificación de sus modalidades han puesto en evidencia lo insuficiente y parcial que resulta la conceptualización del fenómeno como fuga de cerebros. El reconocimiento del carácter complejo y dinámico de la migración calificada permite no sólo captarla en su realidad multifacética, sino también valorar los beneficios 
reales y potenciales de los migrantes de retorno, la movilidad de talentos, la vinculación de la diáspora con la formación de los sistemas nacionales de ciencia y tecnología, el desarrollo empresarial en las áreas de ciencia y tecnología, así como con las necesidades del innovación del aparato productivo (Docquier y Lodigiani, 2010).

Por esta razón, hoy en día se reconoce cada vez más que la migración internacional calificada contribuye a propagar conocimientos y habilidades de innovación de los países receptores hacia los de origen. Sin embargo, conviene señalar que la relación entre la diáspora y el desarrollo de las naciones de origen no es simple sino compleja y dinámica, y depende de una variedad amplia de factores, como el tamaño, composición, nivel de escolaridad y las competencias y destrezas de los migrantes, así como el grado de integración en los países de destino y, por supuesto, las características del entorno económico, político y social (OCDE, 2012). Asimismo, tienen una gran importancia las capacidades de las naciones de origen de vincular a la diáspora con sus sistemas de ciencia y tecnología y las necesidades de innovación.

Por otra parte, debe tomarse en cuenta que existen nuevas condiciones - como el desarrollo de las comunicaciones y los menores costos de los medios transportes - que favorecen no sólo una mayor intensidad de la movilidad internacional de talentos, sino también la multiplicación de contactos con las diásporas.

Este enfoque optimista de la migración internacional calificada ha sido cuestionado tanto porque suele generalizar situaciones particulares exitosas - que pueden limitarse a una nación en un sector o región determinadacomo por la escasa consideración de la lógica de acumulación de capital a escala global, factor estructural que limita la capacidad efectiva de las naciones emergentes de influir e incluso de participar en el proceso de creación y circulación del conocimiento (Cypher y Delgado, 2012).

Sin embargo, cabe señalar que los estudios más recientes y de mayor alcance dejan ver que no es posible formular conclusiones definitivas y válidas para todas las naciones sobre los costos y beneficios de la migración calificada (Beine, Docquier y Rapoport, 2008).

Como bien se ha dicho, el éxito logrado por algunas experiencias de migrantes de países emergentes en sectores de alta tecnología de los países 
avanzados no quiere decir que todas las economías en desarrollo están en condiciones de aprovechar los beneficios de la circulación de cerebros y la promoción de inversiones en sectores de alta tecnología en los países de origen. Esta oportunidad ha sido aprovechada por países que han invertido mucho en la educación superior y son política y económicamente estables para animar a volver a los inmigrantes. ${ }^{13}$

Por consiguiente, para formarse una opinión más informada acerca de los costos y beneficios reales y potenciales que la migración internacional calificada puede tener para los países emisores, es preciso contar con evaluaciones de mediano y largo plazos. México puede y debe aprovechar los hallazgos de las evaluaciones realizadas en otros contextos y contrastarlas con la experiencia local para evitar trasplantar acríticamente las orientaciones y líneas de política. Especialmente, no debe pasarse por alto que México tiene particularidades que obligan a introducir matices a las opiniones optimistas (o pesimistas) que se apoyan en algunas experiencias nacionales o en información fragmentaria referida a algún sector productivo en una coyuntura singular.

Desde el punto de vista de las necesidades de investigación en México, resulta de gran relevancia profundizar en el conocimiento de las distintas modalidades e implicaciones de la migración de personas altamente calificadas, así como impulsar evaluaciones de los programas oficiales destinados a estimular el retorno y diseñar instrumentos adecuados que informen sobre la dinámica particular de los procesos de reintegración de los científicos y tecnólogos (Fernández, 2011; Didou, 2009; Cruz y Ruiz, 2010).

Para el diseño de políticas públicas tiene una enorme importancia el conocimiento de los procesos de toma de decisiones de los migrantes altamente calificados, muy particularmente de las evaluaciones costo-beneficio de permanecer allá o volver al país (Durand, 2005). También es necesario profundizar en el conocimiento de las motivaciones de quienes regresaron a México y deciden volver a Estados Unidos o a otro país avanzado, en lo cual segura-

${ }_{13} \mathrm{Al}$ respecto, se ha dicho que "gran parte de África y América Latina carece de la base de conocimientos o la apertura política para convertirse en entornos atractivos para la iniciativa empresarial tecnológica» (Saxenian, 2005). 
mente influye no sólo el nivel salarial, sino también la infraestructura científica y tecnológica disponible para el desarrollo profesional en los centros de investigación, o las dificultades para insertarse desde México en las redes que vinculan a los investigadores con el flujo del conocimiento global.

Asimismo, se requieren mediciones sistemáticas de la emigración calificada permanente y circular y profundizar en el conocimiento de las trayectorias laborales por grado académico y sector de actividad de los migrantes altamente calificados tanto en México, como en Estados Unidos. También es preciso multiplicar los estudios para valorar las posibilidades de vincular a los migrantes de retorno y a quienes permanecen en el exterior con la formación de capital humano, el sistema nacional de innovación y la estrategia de desarrollo de México.

En este contexto, resulta imperioso desarrollar para México una política multifacética que vaya más allá de los programas de factura clásica dirigidos a prevenir el éxodo definitivo de personal especializado, a alentar su regreso y garantizar su reintegración. Para ello, es necesario diseñar respuestas creativas de política pública a fenómenos como la circulación de talentos, la formación de redes y la emergencia de comunidades científicas virtuales. Asimismo, se requiere tomar en cuenta el punto de vista de quienes ya no pueden o no quieren regresar a México: sus conocimientos, contactos y experiencias siempre podrían representar un activo enorme cuando se movilizan a favor del desarrollo del país.

Sin duda, las políticas de retención, promoción del retorno e impulso a la circulación de talentos exigen una vinculación de la política migratoria con la estrategia nacional de desarrollo. Por sí sola, la política migratoria sería infructuosa. Es imprescindible sumar voluntades y recursos para crear en el país condiciones institucionales y productivas que efectivamente reduzcan la brecha tecnológica y mejoren las condiciones de trabajo (infraestructura y equipamiento) de los científicos y talentos nacionales. A este respecto, es alentadora la decisión del presidente Enrique Peña Nieto de sostener un aumento sistemático de recursos al sistema nacional de ciencia y tecnología, así como alentar los intercambios con Estados Unidos en materia de educación superior.

$$
60 \frac{\text { SEGUNDO SEMESTRE } 2013}{\text { MIGRACIÓN Y DESARROLLO NÚM. } 21}
$$


Una política multifacética en esta materia exige avanzar en muy distintos frentes, con acciones de diferente alcance, pero todas ordenadas en función de las prioridades del desarrollo y del sistema nacional de innovación. En este sentido, es muy relevante promover una cultura empresarial que reconozca el valor de la innovación como fuente de competitividad internacional y de un mayor retorno de las inversiones productivas. La actual proporción de posgraduados contratados en el sector productivo privado es verdaderamente reducida. Asimismo, los centros de investigación y las universidades están llamados a desempeñar un papel aún más relevante en el desarrollo de las estrategias de innovación y ser el vínculo primordial de participación nacional en las redes globales del conocimiento.

En todo caso, el gran reto para México es que la movilidad calificada y de talentos no se traduzca en una pérdida definitiva de recursos humanos, sino en una oportunidad para el desarrollo nacional que abre la globalización.

\section{Bibliografía}

Albo, Adolfo y Juan Luis Ordaz Díaz (2011), Migración mexicana altamente calificada en EE.UU. y transferencia de México a Estados Unidos a través del gasto en la educación de los migrantes, México, BBva Bancomer, Documento de Trabajo número 11/25.

Beine, Michel, Frederic Docquier y Hillel Rapoport (2008), «Brain drain and human capital formation in developing countries: winners and losers", The Economic Journal, 118 (abril), 631-652.

Cenna, Lucie (2010), Policies and practices of highly skilled migration in times of the economic crisis, Ginebra, orT.

Cypher, James y Raúl Delgado (2012), México a la deriva. Génesis, desempeño y crisis del Modelo Exportador de Fuerza de Trabajo, Colección Desarrollo y Migración. México: Estudios Críticos del Desarrollo, uaz, Unesco, Miguel Ángel Porrúa.

Cruz Piñeiro, Rodolfo y Wilfrido Ruiz Ochoa (2010), «Migración calificada de mexicanos a Estados Unidos mediante visado preferencial», Revista Papeles de Población, núm. 66, México, CIEAP/UAEM.

De HAAS, Hein (2010), «Migration and development: a theoretical perspective», International Migration Review, vol. 44 (1). 
Didou, Sylvie (2009), «żPérdida de cerebros y ganancia de saberes?: la movilidad internacional de recursos humanos altamente calificados en América Latina y el Caribe», en Didou, Sylvie y Etienne Gérard (2009) (eds.), Fuga de cerebros, movilidad académica, redes científicas. Perspectivas latinoamericanas, México, IESALC-CINVESTAV-IRD.

Docouier, Frédéric y Hillel Rapoport (2011), «Globalization, brain drain and development», Journal of Economic Literature (en prensa).

y Elisabetta Lodigiani (2010), "Skilled Migration and Business Networks», Open Economies Review, springer, vol. 21(4), septiembre.

Dos Santos, Theotonio (1968), El nuevo carácter de la dependencia, Santiago de Chile, Centro de Estudios Socio-Económicos, Universidad de Chile.

DURAND, Jorge (2005), «Ensayo teórico sobre la migración de retorno: el principio del rendimiento decreciente», en Raúl Delgado Wise y Beatrice Kneer (coords.), Contribuciones al análisis de la migración internacional y el desarrollo regional en México, México, Universidad Autónoma de Zacatecas/Miguel Ángel Porrúa, pp. 309-318.

Emmanuel, Arghiri (1971), «El intercambio desigual», en Imperialismo y comercio internacional. Intercambio desigual, México, Siglo XXI Editores, 1971.

EUR-Lex (2009), «Directiva 2009/50/CE del Consejo, de 25 de mayo de 2009, relativa a las condiciones de entrada y residencia de nacionales de terceros países para fines de empleo altamente cualificado», en http://eur-lex.europa.eu/LexUriServ/LexUriServ.do? uri=CELEX:32009L0050:ES:NOT (consultado el 3 de julio de 2013).

FERNÁNDEZ GUZMÁn, Eduardo (2011), «Revisión bibliográfica sobre la migración de retorno", revista Norteamérica [online], vol. 6, núm. 1, México, CISAN, UnAm, pp. 35-68.

KNeRr, Béatrice (2007), "Crisis económica y migración laboral internacional: costos y beneficios en Alemania», en Migración y Desarrollo, vol. 9, Zacatecas, Primer Semestre, pp. 24-47.

Marmolejo, Francisco (2009), "Redes, movilidad académica y fuga de cerebros en América del Norte: el caso de los académicos mexicanos», en Didou, Sylvie y Etienne Gérard, (eds.), Fuga de cerebros, movilidad académica, redes científicas. Perspectivas latinoamericanas, México, IESALC-CINVESTAV-IRD.

OCDE (2012), Connecting with Emigrants: A Global Profile of Diasporas, OECD, http:// dx.doi.org/10.1787/9789264177949-en.

(2012), Settling In: OECD Indicators of Immigrant Integration 2012, OCDE, http:dx.doi.org/10.1787/9782264171534-en.

$62 \frac{\text { SEGUNDO SEMESTRE } 2013}{\text { MIGRACIÓN Y DESARROLLO NÚM. } 21}$ 
(2009), La competición global por el talento. Movilidad de los trabajadores altamente calificados, España, OCDE-INAP.

OIM (2009), La emigración de recursos humanos calificados desde países de América Latina y el Caribe. Tendencias contemporáneas y perspectivas, Venezuela.

Prebisch, Raúl (1949), "El desarrollo económico de la América Latina y algunos de sus principales problemas», Santiago de Chile, CEPAL. Reproducido en A. Gurrieri, La obra de Prebisch en la CEPAL, México, Fondo de Cultura Económica, 1982. (1981), Capitalismo periférico. Crisis y transformación, México, Fondo de Cultura Económica.

SAXENian, AnnaLee (2005), «From brain drain to brain circulation: Transnational communities and regional upgrading in India and Chinaw, Studies in Comparative International Development, vol. 40, núm. 2.

Stiglitz, Joseph (2012), El precio de la desigualdad, España, Taurus. 
